\title{
Deformation state of short-period AlGaN/GaN superlattices at different well-barrier thickness ratios
}

\author{
V.P. Kladko, N.V. Safriuk, H.V. Stanchu, A.V. Kuchuk, V.P. Melnyk, A.S. Oberemok, S.B. Kriviy, \\ Z.V. Maksymenko, A.E. Belyaev, B.S. Yavich ${ }^{1}$ \\ V. Lashkaryov Institute of Semiconductor Physics, NAS of Ukraine, \\ 41, prospect Nauky, 03028 Kyiv, Ukraine

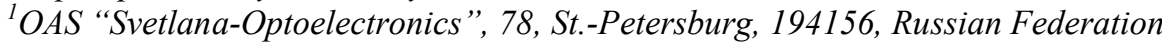

\begin{abstract}
Dependence of deformation characteristics changing in superlattice (SL) structures $\mathrm{Al}_{\mathrm{x}} \mathrm{Ga}_{1-\mathrm{x}} \mathrm{N} / \mathrm{GaN}$ with $\mathrm{Al}(\sim 10 \%)$ on the well-barrier thickness ratio in period was studied in this work. The deformation state of SL and individual layers, relaxation level and periods, layers' thickness and composition of $\mathrm{Al}_{\mathrm{x}} \mathrm{Ga}_{1-\mathrm{x}} \mathrm{N}$ layers were analyzed using high-resolution X-ray diffractometry. It was ascertained that the buffer layer and SL layers are compressed in all the investigated structures. Thus, it has been shown that deformation of the SL period depends on the well/barrier thickness ratio. Thicknesses of individual layers in SL strongly depend on the deformation state of the whole system. Increasing the deformation level leads to the increase of the barrier layer thickness.
\end{abstract}

Keywords: superlattice structure, gallium nitride, X-ray diffractometry, deformation characteristics.

Manuscript received 23.06.14; revised version received 14.08.14; accepted for publication 29.10.14; published online 10.11.14.

\section{Introduction}

Multilayered structures based on alloys $\mathrm{Al}_{\mathrm{x}} \mathrm{Ga}_{1-\mathrm{x}} \mathrm{N} / \mathrm{GaN}$, the so-called superlattices (SL), are widely used for light emitting and laser diodes in UV and visible spectral ranges as well as for high-power and high-temperature field-effect transistors [1,2]. These structures were thoroughly investigated for the latter ten years by various groups of researchers.

Despite many efforts to grow these structures of high quality with specified properties they still are failed. In previous works [3-6], it was shown that at epitaxial growth of nitride structures they relaxed by formation of dislocations and other defects also by changing of well-barrier thicknesses in SL from technological thicknesses. The deformation state, thickness fluctuation and defects in SL negatively affect on the devices performance changing their wavelength, carrier transport and carrier lifetime [7-9]. Intrinsic electrostatic fields in nitride structures are an important factor that influences the optical properties of these structures, they leads to huge polarization, roughness of surface and interfaces $\mathrm{Al}_{\mathrm{x}} \mathrm{Ga}_{1-\mathrm{x}} \mathrm{N} / \mathrm{GaN}$. These fields strongly depend on the deformation state $[10,11]$. As it was shown previously [12], deformation in $\mathrm{Al}_{\mathrm{x}} \mathrm{Ga}_{1-\mathrm{x}} \mathrm{N} / \mathrm{GaN} \mathrm{SL}$ can cause the shift of irradiation area changing its electron properties. Structures of IIInitrides have high piezoelectric constant in $\langle 0001\rangle$ direction. Strain in SL layers leads to increasing of piezoelectric fields, which changes the potential profile and cause the red shift of emission well known as the Stark effect. 
Taking into account previous facts, investigation of the deformation state and variation of the layer thickness is very important for optimization of nitride structure growth.

High-resolution X-ray diffractometry (HRXD) is one of nondestructive diagnostic methods widely used to determine structural parameters of multilayered structures such as composition, thickness of appropriate layers, SL period, interface sharpness (existence of transitional layers), deformation in layers and type of defects $[13,14]$.

The reasons of origin and relaxation of mechanic strain in $\mathrm{AlGaN}$ and $\mathrm{GaN}$ layers of SL at different ratios of these thicknesses were investigated in this work. The investigated samples were test samples with AlGaN layers parameters for designing blue light diodes. We study dependence of structure parameters on the growth regime. In turn, parameters of nanosized layers are more convenient to be defined in SL. All the values were obtained from very precise measurements by using HRXD, X-ray reflectometry and secondary neutral mass-spectrometry (SNMS).

\section{Theory}

As usual, III-nitride films grown on the sapphire substrate are fully relaxed at the growth temperature, and their deformations at room temperature have mostly a thermal character. Taking into account this fact, one can describe relaxation by two parameters - relaxation of SL with respect to the substrate and relaxation of each layer with respect to other layers in SL.

For wurtzite structures (typical for $\mathrm{AlGaN}$ and $\mathrm{GaN})$ that grow along hexagonal axes $\langle 0001\rangle$, the lattice parameter $a$ defines the interplanar distance in the interface plane, while the lattice parameter $c$ - in the direction perpendicular to the interface. Let us denote real (measured) lattice parameters as $a_{i}$ and $c_{i}$ for each $i$ layer, $a_{i}^{b}$ and $c_{i}^{b}$-corresponding values for the layer in unstrained state, where the index $i=0$ corresponds to the buffer layer, indexes $i=1,2$ correspond to AlGaN and GaN SL's sub-layers. Elastic deformation in SL layers is given by:

$\varepsilon_{i}=\frac{a_{i}-a_{i}^{b}}{a_{i}^{b}}$.

The real parameter $c_{i}=c_{i}^{b}\left(1-p \varepsilon_{i}\right)$, where $p=2 c_{13} / c_{33}$ is the Poisson ratio. Thus, strain relaxation in SL can be characterized by deformation jump $\Delta a_{i}=a_{i}-a_{i-1}$ of lattice parameter $a$ at the interface, i.e., the so-called relaxation level:

$r_{i}=\frac{a_{i}-a_{i-1}}{a_{i}^{b}-a_{i-1}}=\frac{\Delta a_{i}}{a_{i}^{b}-a_{i-1}}$.
The parameters $\Delta a_{1}$ and $r_{1}$ correspond to relaxation on the bottom interface (between the buffer layer and first layer of SL), parameters $\Delta a_{2}$ and $r_{2}$ - relaxation at the interfaces of subsequent layers (Fig. 1). For a strained coherent structure $\Delta a_{2}=\Delta a_{1}=0$. When subsequent layers grow coherently and relax with respect to the buffer layer as a whole $\Delta a_{2}=0, \Delta a_{1}$ can be higher or lower than 0 , which depends on the composition of the buffer layer. In general, in the case of relaxed incoherent SL both jumps can be no equal to zero, at the same time $\Delta a_{2}$ must be equal at all interfaces in SL.

Lattice parameters' mismatch in SL can be obtained using the equation:

$\frac{\Delta a}{\langle a\rangle}=\frac{a_{\mathrm{GaN}}^{b}-a_{\mathrm{AlGaN}}^{b}}{\langle a\rangle}$,

where $\langle a\rangle=\left(a_{\mathrm{GaN}}^{b}+a_{\mathrm{AlGaN}}^{b}\right) / 2$.

The average lattice parameter $a$ in SL is equal:

$\left\langle a_{S L}\right\rangle=\frac{t_{\mathrm{GaN}} \cdot a_{\mathrm{GaN}}^{b}+t_{\mathrm{AlGaN}} \cdot a_{\mathrm{AlGaN}}^{b}}{t_{\mathrm{GaN}}+t_{\mathrm{AlGaN}}}$.

In case of symmetrical SL $\left(t_{\mathrm{GaN}}=t_{\mathrm{AlGaN}}\right)$, parameters are equal $\left\langle a_{S L}\right\rangle=\langle a\rangle$.

For SL AlGaN/GaN, which growth is pseudomorph to that of the buffer layer, the jumps of lattice parameters are $\Delta a_{1}=0$ and $\Delta a_{2}=0$ between layers in SL and between buffer layer and SL, correspondingly. In this case, deformations in $\mathrm{AlGaN}$ and $\mathrm{GaN}$ layers can be described as:

$\varepsilon_{\mathrm{GaN}}=0 ; \quad \varepsilon_{\mathrm{AlGaN}}=-\frac{\Delta a}{\langle a\rangle}$.

The average lattice parameter $a$ in SL period is equal:

$\left\langle\varepsilon_{S L}\right\rangle=\frac{\varepsilon_{\mathrm{GaN}} \cdot t_{\mathrm{GaN}}+\varepsilon_{\mathrm{AlGaN}} \cdot t_{\mathrm{AlGaN}}}{t_{\mathrm{GaN}}+t_{\mathrm{AlGaN}}}=$

$=\frac{\varepsilon_{\mathrm{AlGaN}} \cdot t_{\mathrm{AlGaN}}}{t_{\mathrm{GaN}}+t_{\mathrm{AlGaN}}}=-\frac{\Delta a}{\langle a\rangle} \cdot \frac{t_{\mathrm{AlGaN}}}{T}$,

here $T=t_{\mathrm{GaN}}+t_{\mathrm{AlGaN}}$. When SL is partly relaxed, we must use both parameters of relaxation. The first parameter $\Delta a_{1}=a_{\mathrm{GaN}}-a_{\mathrm{AlGaN}}$ is a jump of the lattice parameter between individual layers $\mathrm{AlN}$ and $\mathrm{GaN}$ in SL. These jumps must be equal on all the interfaces, if relaxation doesn't change periodicity of SL. Another parameter $\Delta a_{2}$ is the jump of the lattice parameter $a$ at the interface buffer - first layer of SL. This parameter describes relaxation of SL.

Lateral deformation in $\mathrm{AlGaN}$ and GaN layers and average deformation in SL period are given by: 
$\varepsilon_{\mathrm{AlGaN}}=-\frac{\Delta a}{\langle a\rangle}+\frac{\Delta a_{1}}{\langle a\rangle}=-\frac{1}{\langle a\rangle}\left(\Delta a-\Delta a_{1}\right)$,

$\left\langle\varepsilon_{S L}\right\rangle=-\frac{\left(\Delta a-\Delta a_{1}\right)}{\langle a\rangle} \cdot \frac{t_{\mathrm{AlGaN}}}{T}$.

Average deformation in SL period is $\left\langle\varepsilon_{S L}\right\rangle=-\left(\Delta a-\Delta a^{\prime}\right) / 2\langle a\rangle$, when layers AlN and GaN in SL have equal thicknesses. As soon as deformation in SL reach a critical value when thickness increases, there appears situation when SL relaxes as a whole with the deformation jump $\Delta a^{\prime \prime}$. This relaxation (partly in general) leads to average lateral deformation:

$\left\langle\varepsilon_{S L}^{\prime}\right\rangle=k \cdot \frac{\Delta a}{\langle a\rangle} \cdot \frac{t_{\mathrm{AlGaN}}}{T}=k \cdot \frac{\left(\Delta a-\Delta a_{2}\right)}{\langle a\rangle} \cdot \frac{t_{\mathrm{AlGaN}}}{T}$,

where the coefficient $k$ is $-1 \leq k \leq 1$. In case when $k=0$, SL is fully relaxed, while $k=-1$ and 1 describe the cases when layers of SL have lateral parameters of AlN or GaN buffer layers, respectively. Three parameters of relaxation are related as:

$\frac{\Delta a}{\langle a\rangle} \cdot \frac{t_{\mathrm{AlGaN}}}{T}(1+k)=\frac{\Delta a^{\prime}}{\langle a\rangle}+\frac{\Delta a^{\prime \prime}}{\langle a\rangle}$.

Relaxation of SL as a whole leads to changes of the average SL parameters and, correspondly, to the shift of the diffraction picture (curve) with respect to the SL peak. But this relaxation doesn't change the intensity ratio of the satellites. Relaxation between layers leads to changes in deformation $\varepsilon_{\perp}$, which can be given as:

$$
\begin{aligned}
& \Delta \varepsilon_{\perp}=\frac{c_{\mathrm{AlGaN}}-c_{\mathrm{GaN}}}{c_{\mathrm{GaN}}} x+ \\
& +p \frac{a_{\mathrm{AlGaN}}-a_{\mathrm{GaN}}}{a_{\mathrm{GaN}}} x-p \frac{\Delta a_{2}}{a_{\mathrm{GaN}}},
\end{aligned}
$$

where $c_{\mathrm{GaN}}, a_{\mathrm{GaN}}$ and $c_{\mathrm{AlGaN}}, a_{\mathrm{AlGaN}}$ are table lattice parameters of $\mathrm{GaN}$ and $\mathrm{AlGaN}$, respectively. The Poisson ratio $p=2 c_{13} / c_{33}$ is equal for $\mathrm{GaN}$ and $\mathrm{AlGaN}$ layers.

\section{Experimental}

10-period $\mathrm{Al}_{\mathrm{x}} \mathrm{Ga}_{1-\mathrm{x}} \mathrm{N} / \mathrm{GaN}$ SL obtained by MOCVD were investigated in this work. SL structures were grown on complex GaN-buffer layer that contain GaN:Si $(3.5 \mu \mathrm{m})$ layer, $\mathrm{GaN}$ layer with intrinsic conductivity $(0.5 \mu \mathrm{m})$ and low-temperature nucleated $\mathrm{GaN}$ layer $(20 \mathrm{~nm})$ grown on $c$-plane of sapphire. The first series of SL (S1) consisted of barriers $\mathrm{Al}_{\mathrm{x}} \mathrm{Ga}_{1-\mathrm{x}} \mathrm{N}$ with the nominal thickness close to $6.6 \mathrm{~nm}$ with nominal composition $\mathrm{Al} \sim 10 \%$ and $\mathrm{GaN}$ well with the nominal thickness near $9 \mathrm{~nm}$. In the second series (S2) the $\mathrm{Al}_{\mathrm{x}} \mathrm{Ga}_{1-\mathrm{x}} \mathrm{N}$ nominal thickness of barriers was approximately $10 \mathrm{~nm}$, the GaN well thickness was close to $6 \mathrm{~nm}$. Thickness values were calculated from the growth time of SL layers.

Investigation of the samples was performed using high-relosution X-ray diffractometer PANalytical X'Pert PRO MRD. The reciprocal space map (RSM) and diffraction curves (DC) obtained in triple-axis scheme were used to analyze structural parameters. For calculation of deformation in the buffer layer $\mathrm{GaN}$ experimental DC were normalized to 0006 reflection of the sapphire substrate at $2 \theta=41.680^{\circ}$. Theoretical DC were calculated using the plane wave methods $[15,16]$. Ideal structural parameters for all the layers were taken from the papers $[17,18]$. Dislocation density measurements were performed like to those in works $[19,20]$. The thickness of individual layers in SL and its period were controlled using X-ray reflectivity and secondary neutral mass-spectrometry (SNMS).

The layer-by-layer depth analysis of dopant distribution in $\mathrm{Al}_{\mathrm{x}} \mathrm{Ga}_{1-\mathrm{x}} \mathrm{N} / \mathrm{GaN}$ SL was carried out using SNMS. Measurements were performed in highfrequency (HF) regime of sputtering the sample by $\mathrm{Ar}^{+}$ ions with $330 \mathrm{eV}$ in INA-3 (Laybold-Heraeus) equipment. Ions were generated due to the application of HF voltage in the form of rectangular pulses between samples and wall of $\mathrm{HF}$ plasma at low pressure $\left(3.26 \cdot 10^{-2}\right.$ mbar ). The voltage frequency was $50 \mathrm{kHz}$. The area of sputtering was limited by the tantal diaphragm with the internal diameter $3 \mathrm{~mm}$. Selected parameters of sputtering allowed to provide step-by-step analysis with high resolution $\sim 1 \mathrm{~nm}$ for the depth $100 \mathrm{~nm}$. The dispersion speed of $\mathrm{AlGaN}$ and GaN layers was estimated from the ratio between the crater etching depth and dispersion time for each layer. The crater etching depth was obtained using atomic force microscopy (AFM) (NanoScope IIIa Dimension 3000). The crater etching depth and thickness of individual layers were determined using the methods from the work [21].

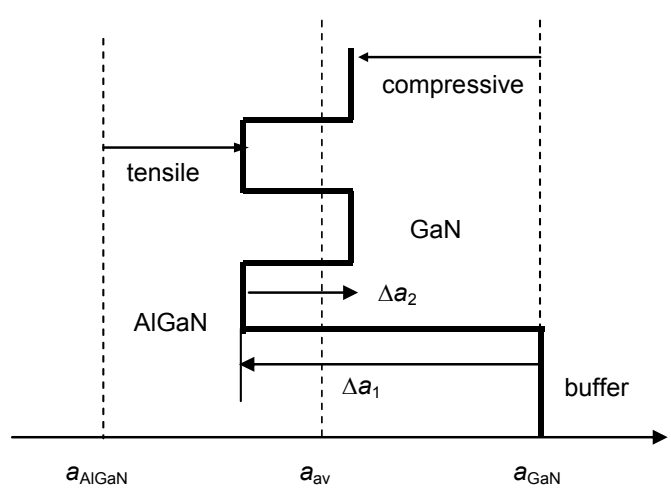

Fig. 1. Distribution of the lattice parameter $a$ along the depth of SL $\mathrm{AlGaN} / \mathrm{GaN}$ grown on the buffer layer GaN. The dashed layer corresponds to the parameter $a$ for unstrained layers and average parameter over SL. 


\section{Results}

Series of RSM in the vicinity of reflexes $(11 \overline{2} 4)$ and $(10 \overline{1} 5)$ for all structures were analyzed. RSM for $(11 \overline{2} 4)$ reflex of both structures S1 and S2 is presented in Fig. 2. Information about the relaxation level in heterostructures can be obtained from asymmetric RSM where the diffraction vector makes the angle $\varphi$ with surface. The intensity of coherent distribution for additional nods (from individual layers, thickness oscillations and SL satellites) for the fully relaxed structure lay in the diffraction plane parallel to the surface normal [20]. Such growth must be provided to obtain structures suitable for application in devices. For the fully relaxed samples, diffraction nods are placed along the diffraction vector. When they are partly relaxed, nods are located in an intermediate position.

Thus, in case when intensity distribution from SL and substrate are placed on the surface normal, it shows coherent interface, on the other hand, another placement of intensity distribution corresponds to some relaxation level of SL with respect to the substrate [20].

A small shift between SL satellites and buffer layer in the vertical direction, which indicates partial relaxation at this boundary $\left(\Delta a_{1} \neq 0\right)$, was observed in our work. Moreover, for S1 sample relaxation level is higher $\left(r_{1}=0.047\right)$ than for the sample S2 $\left(r_{1}=0.037\right)$.

Interesting fact was noticed from RSM: peak position of $\mathrm{GaN}$ indicates a compressed state. Thus, the buffer layer wasn't fully relaxed. The average lattice parameter $a$ of SL is bigger than that parameter of the buffer layer. It indicates that pseudomorphic growth of SL is corrupted and appearance of satellites shows the coherent growth.

Broad intensity distribution from the buffer layer $\mathrm{GaN}$ and satellites from SL $\mathrm{Al}_{\mathrm{x}} \mathrm{Ga}_{1-\mathrm{x}} \mathrm{N} / \mathrm{GaN}$ is depicted in Fig. 2. As one can see from this figure, intensity maxima from the buffer layer $\mathrm{GaN}$ are wider in $Q_{x}$ direction, which indicates presence of defects (point defects, dislocations). It is well known that the epitaxial layer of III-nitride is often grown on a sapphire substrate and has a high dislocation density of threading dislocation $\left(\sim 10^{9} \mathrm{~cm}^{-2}\right)$, which leads to broadening of diffraction maxima in the direction parallel to surface $[21,22]$. In our case, the dislocation density in $\mathrm{Al}_{\mathrm{x}} \mathrm{Ga}_{1-\mathrm{x}} \mathrm{N} / \mathrm{GaN}$ SL is extremely lower $\left(\sim 10^{7} \mathrm{~cm}^{-2}\right)$. Also, from analysis of Fig. 2 one can observe broadening of satellites in direction along the diffraction
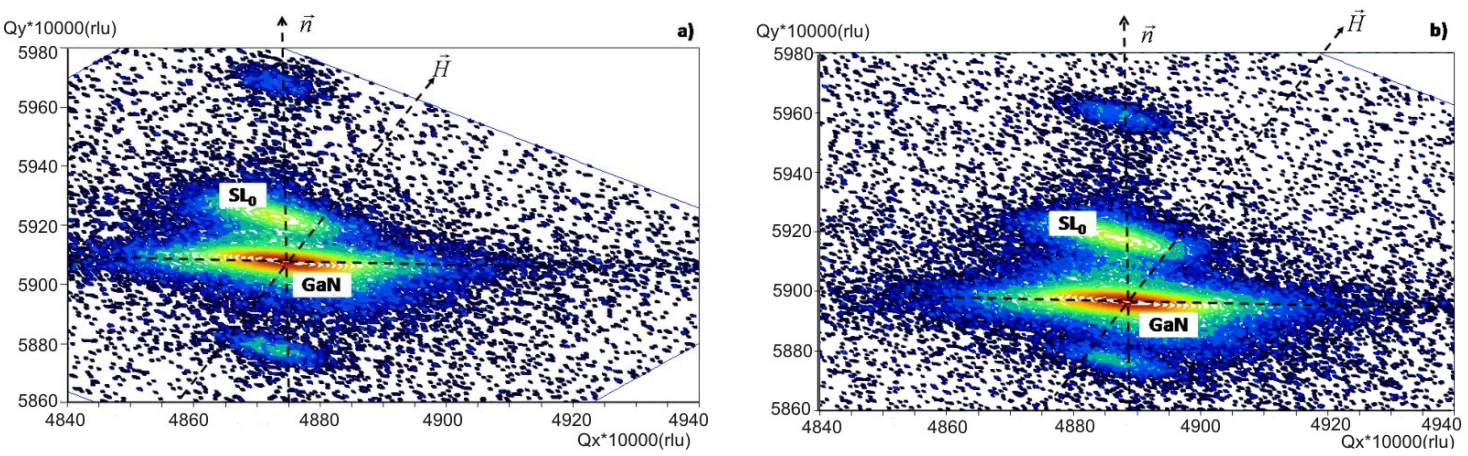

Fig. 2. RSM $(11 \overline{2} 4)$ for SL AlGaN/GaN: S1 $(a), \mathrm{S} 2(b) . Q_{y}$ and $Q_{x}$ are reciprocal space coordinates perpendicular and parallel to the surface, respectively. $\vec{H}$ is the diffraction vector, $\vec{n}$ - surface normal vector, $\mathrm{SL}_{0}$ - position of $\mathrm{SL}_{0}$ satellite.
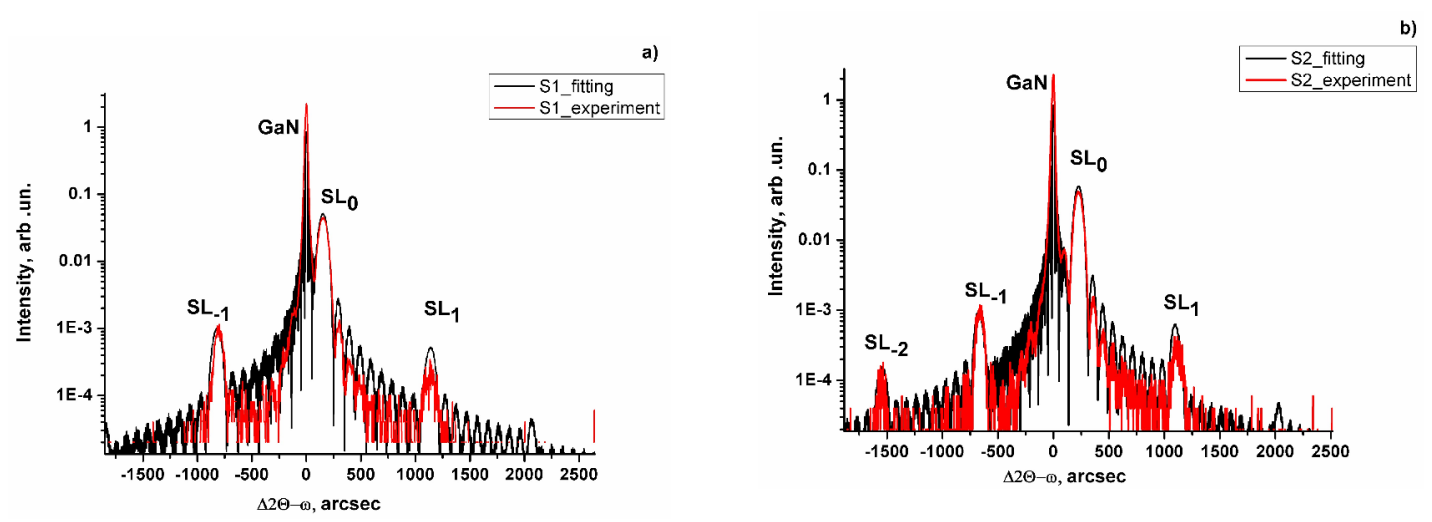

Fig. 3. $\omega-2 \theta$-scans for symmetrical reflex 0002 from SL AlGaN/GaN: a) S1, b) S2. Experiment - lower (red) curve, fittingupper (black) curve. $\mathrm{SL}_{n}-$ satellites of SL. 
Table 1. Technological (nominal) and experimental parameters of $\mathrm{Al}_{\mathbf{x}} \mathrm{Ga}_{1-\mathrm{x}} \mathrm{N} / \mathrm{GaN}$.

\begin{tabular}{|c|c|c|c|c|c|c|c|c|}
\hline Sample & $\begin{array}{c}\text { Layers of } \\
\text { SL }\end{array}$ & $\begin{array}{c}T_{\text {nomin }}, \\
\mathrm{nm}\end{array}$ & $\begin{array}{c}t_{\mathrm{XRD}}, \\
\mathrm{nm}\end{array}$ & $\begin{array}{c}t_{\mathrm{SIMS}} \\
\mathrm{nm}\end{array}$ & $T_{\text {nomin }} / \mathrm{T}_{\mathrm{XRD}} / T_{\text {refl }}, \mathrm{nm}$ & $x_{\mathrm{SIMS}} / x_{\mathrm{XRD}}, \%$ & $\begin{array}{c}N_{s}, \\
\times 10^{7} \mathrm{~cm}^{-2}\end{array}$ & $\begin{array}{l}R_{\text {curv }}, \\
\mathrm{m}\end{array}$ \\
\hline \multirow{2}{*}{ S1 } & $\mathrm{GaN}$ & 9 & 10 & 10 & \multirow{2}{*}{$15.6 / 17 / 17.2$} & \multirow{2}{*}{$9 / 10$} & \multirow{2}{*}{8.37} & \multirow{2}{*}{6.8} \\
\hline & $\mathrm{AlGaN}$ & 6.6 & 7 & 8 & & & & \\
\hline \multirow{2}{*}{ S2 } & $\mathrm{GaN}$ & 6 & 5.5 & 6.5 & \multirow{2}{*}{$16 / 18.7 / 18$} & \multirow{2}{*}{$10 / 10$} & \multirow{2}{*}{6.00} & \multirow{2}{*}{6.6} \\
\hline & $\mathrm{AlGaN}$ & 10 & 13.2 & 12.66 & & & & \\
\hline
\end{tabular}

Table 2. Lattice parameters of the buffer layer and SL: ideal and calculated values.

\begin{tabular}{|c|c|c|c|c|c|c|c|c|}
\hline $\begin{array}{l}\stackrel{0}{\tilde{\Xi}} \\
\text { 壹 }\end{array}$ & $a_{S L}^{i d}, \mathrm{~nm}$ & $c_{S L}^{i d}, \mathrm{~nm}$ & $a_{\mathrm{GaN}}^{i d}, \mathrm{~nm}$ & $c_{\mathrm{GaN}}^{i d}, \mathrm{~nm}$ & $\left\langle a_{S L}\right\rangle, \mathrm{nm}$ & $\left\langle c_{S L}\right\rangle, \mathrm{nm}$ & $a_{\mathrm{GaN}}^{b u f}, \mathrm{~nm}$ & $c_{\mathrm{GaN}}^{b u f}, \mathrm{~nm}$ \\
\hline $\mathrm{S} 1$ & 0.318598 & 0.51767 & 0.31892 & 0.51850 & 0.316422 & 0.520625 & 0.316314 & 0.521985 \\
\hline $\mathrm{S} 2$ & 0.318368 & 0.51708 & 0.31892 & 0.51850 & 0.315526 & 0.521079 & 0.315417 & 0.522979 \\
\hline
\end{tabular}

vector. This indicates a stronger effect of mosaicity in SL than in the buffer layer.

Modeling of experimental DC for the symmetrical reflex 0002, being based on the dynamical diffraction theory, was used for a more precise analysis of $\mathrm{Al}_{\mathrm{x}} \mathrm{Ga}_{1-\mathrm{x}} \mathrm{N} / \mathrm{GaN}$ parameters (Fig. 3) [11]. In Fig. 3, the sharp peak corresponds for 0002 reflex from the buffer layer $\mathrm{GaN}$, broader peaks (satellites) corresponds to SL $\mathrm{Al}_{\mathrm{x}} \mathrm{Ga}_{1-\mathrm{x}} \mathrm{N} / \mathrm{GaN}$. The peak position of SL depends on both composition of SL and the ratio of layer thickness.

$\delta \theta$ is the distance between satellites, which marks both $\mathrm{SL}_{-1}$ and $\mathrm{SL}_{0}$, corresponds to the $\mathrm{SL}$ period $(T=$ $t_{\text {well }}+t_{\text {barrier }}=\left|\gamma_{h}\right| \lambda / \sin \left(2 \theta_{\mathrm{B}}\right) \delta \theta$, where $\lambda$ is the X-ray wavelength, $\gamma_{h}$ - directing cosines of the X-ray beam, more frequent fringes are caused be the total thickness of SL. The relative intensity of the satellites was used to obtain the thickness ratio, and then the composition of solid solution $\mathrm{Al}_{\mathrm{x}} \mathrm{Ga}_{1-\mathrm{x}} \mathrm{N}$ [23]. Parameters of SL were obtained from RSM and refined by a procedure of fitting experimental and calculated DC $[14,15]$. They are given in Table 1.

Vertical lattice parameters $c$ of buffer layers GaN were calculated from relative changes of the peak position between the buffer layer $\mathrm{GaN}$ (0002) and sapphire substrate (0006). The sapphire peak height was used as a reference value. The parameters $c$ from DC were $5.1856 \AA$ for $\mathrm{S} 1$ sample and $5.1859 \AA$ for $\mathrm{S} 2$ sample. These values were used for calculation of the perpendicular strain (perpendicular to the growth plane) $\varepsilon_{z z}$ by using the formula:

$\varepsilon_{z z}=\left(c-c_{0}\right) / c_{0}$,

where $c_{0}=5.1855 \AA$ lattice parameter for unstrained layer GaN [18]. Perpendicular strain has tensile character as for S1 $\varepsilon_{z z}=1.9 \cdot 10^{-5}$ as for S2 $\varepsilon_{z z}=$ $7.7 \cdot 10^{-5}$, respectively. The parallel strain was calculated using the following relation:

$\varepsilon_{x x}=\left(a-a_{0}\right) / a_{0}$,

where $a_{0}=3.1891 \AA$. The lattice parameters $a$ were obtained from asymmetric scans for $10 \overline{1} 5$ and $11 \overline{2} 4$ reflections, $a=3.1631 \AA$ and $a=3.1542 \AA$ for $\mathrm{S} 1$ and $\mathrm{S} 2$, respectively. The parallel compressing strains are $\varepsilon_{x x}$ $=-8.1 \cdot 10^{-3}$ for $\mathrm{S} 1$ and $\varepsilon_{x x}=-1.1 \cdot 10^{-2}$ for $\mathrm{S} 2$. Let us note that the initial parameters for the buffer layer GaN were the same for all the structures.

SNMS depth profiles of $\mathrm{Al}$ and $\mathrm{Ga}$ elements in $\mathrm{Al}_{\mathrm{x}} \mathrm{Ga}_{1-\mathrm{x}} \mathrm{N} / \mathrm{GaN} \mathrm{SL}$ within the upper three periods for $\mathrm{S} 1$ and S2 samples are presented in Fig. 4. We observed the increase of the GaN well layer thickness by $0.5 \mathrm{~nm}$ for both samples. In both samples, the $\mathrm{Al}_{\mathrm{x}} \mathrm{Ga}_{1-\mathrm{x}} \mathrm{N}$ barrier layer thickness is different, with deviation from nominal value of 0.5 to $1.5 \mathrm{~nm}$. The barrier layer thickness deviation with depth was observed to be equivalent in each period for S1. On the other hand, for $\mathrm{S} 2$ we observed reducing the barrier layer thickness in each dipper period. All the thickness values are presented in Table 1.

\section{Discussion}

$\mathrm{Al}_{\mathrm{x}} \mathrm{Ga}_{1-\mathrm{x}} \mathrm{N} / \mathrm{GaN}$ SL were grown on the buffer layer GaN, which are in the compressed state that is partly relaxed. The difference between the buffer GaN and average SL lattice parameters $a$ indicates partial relaxation on interface buffer layer - SL, i.e. relaxation on the bottom interface, which was observed for all SL. The calculated 


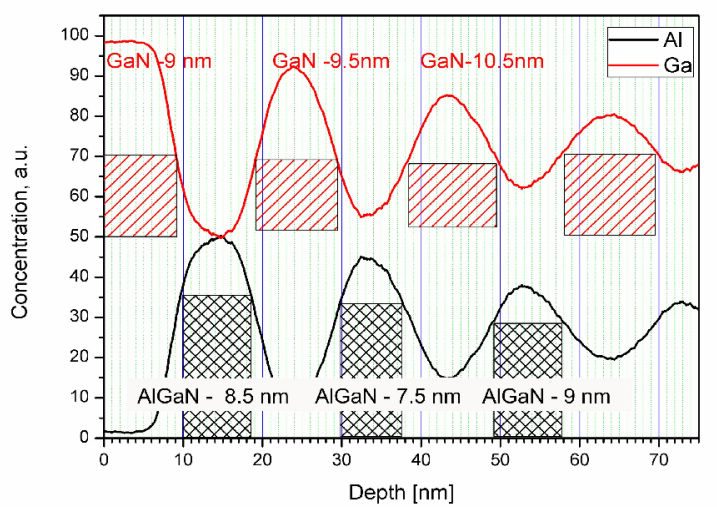

b)

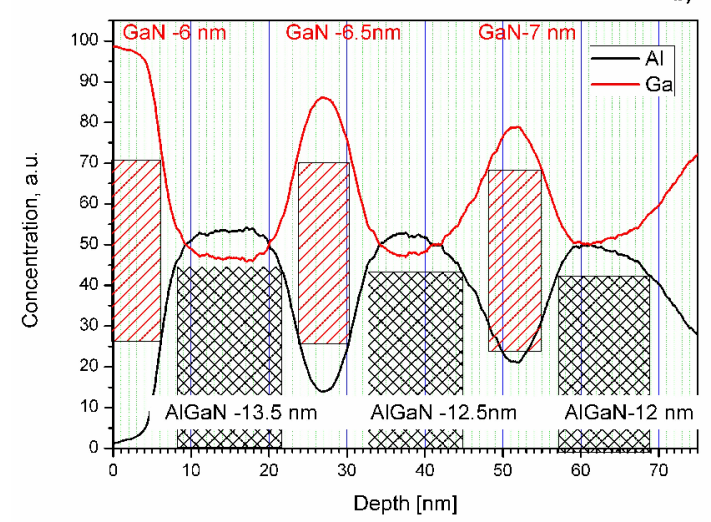

Fig. 4. SNMS depth profiles of $\mathrm{Al}$ and Ga elements in $\mathrm{Al}_{\mathrm{x}} \mathrm{Ga}_{1-\mathrm{x}} \mathrm{N} / \mathrm{GaN}$ SL: a) S1, b) S2. Upper (red) curves - Ga distribution, lower (black) curves - Al distribution.

lattice parameters obtained from asymmetric RSM $(11 \overline{2} 4)$ are summarized in Table 2. From these results, both SL are compressed, but the strain is less than that in the buffer layer. Difference of average SL lattice parameter for S1 and S2 caused by changes in the layer thickness ratio. It influences on the strain state of the buffer and whole system.

As it follows from these results, GaN wells as well as the barrier $\mathrm{Al}_{\mathrm{x}} \mathrm{Ga}_{1-\mathrm{x}} \mathrm{N}$ in SL are in the compressed state $\left(\varepsilon_{x x}<0\right)$ for all the structures (Table 2). The buffer layer also is in the compressed state and leads to the respective state of SL. But in SL this deformation is bigger in well GaN than in the barrier $\mathrm{Al}_{\mathrm{x}} \mathrm{Ga}_{1-\mathrm{x}} \mathrm{N}$. However, the compressing strain is less for $\mathrm{S} 1$ than for S2, which is caused by the different thickness ratio (well-barrier). This behavior in the SL period can be explained from determination of average parameters. Average lattice parameters in the SL period were calculated from the equations (1)-(6). From these equations, one can see that those SL periods are strongly dependent on the thickness ratio well-barrier and on barrier composition.

Deformation profiles with depth of SL calculated with respect to the ideal values of $\mathrm{GaN}$ and $\mathrm{Al}_{\mathrm{x}} \mathrm{Ga}_{1-\mathrm{x}} \mathrm{N}$ are presented in Fig. 5.

The parameter of each SL layer tends to reach the average SL parameter SL0_real, since it tends to reach the real parameter value of the buffer layer GaN (Fig. 5).

Layers in S2 are more strained with respect to the average SL parameter than those in S1. It well correlates with broadening of RSM (0.0064 rlu and $0.0048 \mathrm{rlu}$, for S1 and S2, respectively (Fig. 1)) as well as with the dislocation density that is less in the more strained structure S2.

The strain level of whole SL with respect to buffer layer depends on type of SL. Relaxation is slower for SL with a thick well because of the smaller lattice parameter mismatch between SL and GaN buffer. Compression of the buffer layer affects the strain level in the SL layer and average lattice parameters in the period. But deformation jump at the interfaces of SL layer remains the same, only weak changes of the ratio of strain between the SL layers were observed.

The difference of parameters $\Delta t=t_{\text {exp }}-t_{\text {techn }}$ corresponding to the difference between technological and experimental layer thicknesses are probably caused by different deformation state of the system. As one can see from Fig. 6 for the $\mathrm{Al}_{\mathrm{x}} \mathrm{Ga}_{1-\mathrm{x}} \mathrm{N}$ barrier, the increase of deformation leads to enhancement of the growth rate, on the other hand, we observed the opposite process in the GaN layer. The same changes in the growth rate for the $\mathrm{Al}_{\mathrm{x}} \mathrm{Ga}_{1-\mathrm{x}} \mathrm{N}$ barrier under reducing of mechanical strains were observed in [24]. Explanation of thickness changes with those of deformation was deduced from the first principles for $\mathrm{Al}_{\mathrm{x}} \mathrm{Ga}_{1-\mathrm{x}} \mathrm{N} / \mathrm{GaN}$ SL [25].

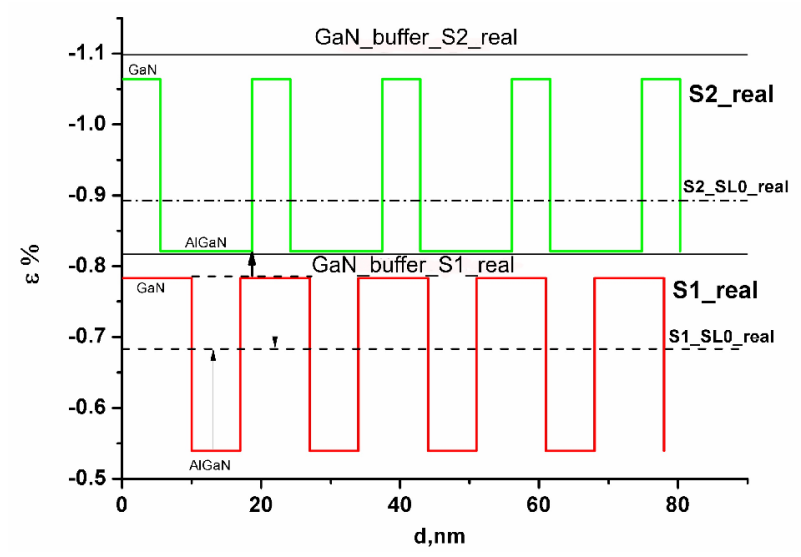

Fig. 5. Deformation profiles alternating with depth of SL in S1 and S2 samples. 


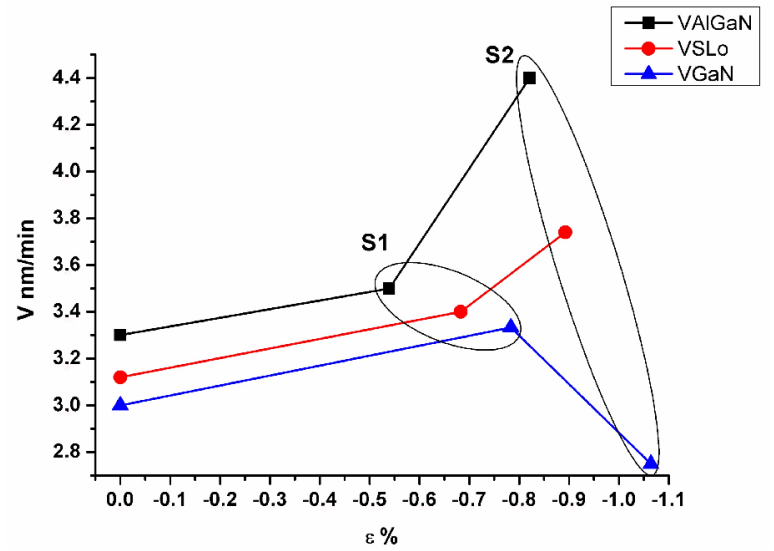

Fig. 6. Dependence of the growth rate $V$ of SL layers $\mathrm{Al}_{\mathrm{x}} \mathrm{Ga}_{1-\mathrm{x}} \mathrm{N}$ and $\mathrm{GaN}$ on the deformation level.

As it follows from Table 1, deformation changes in SL do not lead to significant changes in the dislocation density $N_{s}$ and curvature radius $R_{\text {curv }}$ for each sample. Thus, at the practically equal curvature radius and small deviation of dislocation density we observed different relaxation states for S1 and S2 samples. But, to explain this difference in the relaxation state only due to formation of dislocation is impossible. It indicates that thickness changes of $\mathrm{Al}_{\mathrm{x}} \mathrm{Ga}_{1-\mathrm{x}} \mathrm{N}$ and $\mathrm{GaN}$ layers can be additional relaxation channel in these SLs. Moreover, the process of relaxation and formation of defects are strongly interrelated [26].

\section{Conclusions}

The deformation state of short-period SL and its individual layers, relaxation state, period and layer thickness, composition of $\mathrm{Al}_{\mathrm{x}} \mathrm{Ga}_{1-\mathrm{x}} \mathrm{N}$ were obtained using the X-ray diffraction methods.

It was ascertained that the buffer layer as well as SL layers are compressed in all the investigated structures. Thus, it was shown that deformation of SL period depends on the well-barrier thickness ratio. It, to some extent, determines the relaxation state of whole SL with respect to the buffer layer. However, relaxation state of buffer layer strongly affects deformation of the whole system. The dependence between the growth rate of individual layer and deformation state has been shown. The increase in deformation leads to enhancement of the growth rate for the barrier.

Thus, structural, optical and electro-physical properties of SL are determined by the process of elastic strain relaxation that leads to changing the well and barrier thickness.

This study was supported by the National Academy of Sciences of Ukraine within the framework of the scientific-technological programs "Nanotechnology and Nanomaterials” №3.5.1.12 and №3.5.1.30.

\section{References}

1. H. Morkoç, Handbook of Nitride Semiconductors and Devices: Electronic and Optical Processes in Nitrides. Berlin, Wiley-VCH, 2008.

2. P. Ruterana, M. Albrecht, J. Neugebauer, Nitride Semiconductors: Handbook on Materials and Devices. Berlin, Wiley-VCH, 2003.

3. V.P. Kladko, S.V. Chornen'kii, A.V. Naumov, A.V. Komarov, M. Tacano, Yu.N. Sveshnikov, S.A. Vitusevich, and A.E. Belyaev, Interface structural defects and photoluminescence properties of epitaxial $\mathrm{GaN}$ and $\mathrm{AlGaN} / \mathrm{GaN}$ layers grown on sapphire // Semiconductors, 40, p. 1060 (2006).

4. V.P. Kladko, A.F. Kolomys, M.V. Slobodian, V.V. Strelchuk, V.G. Raycheva, A.E. Belyaev, S.S. Bukalov, H. Hardtdegen, V.A. Sydoruk, N. Klein, and S.A. Vitusevich, Internal strains and crystal structure of the layers in $\mathrm{AlGaN} / \mathrm{GaN}$ heterostructures grown on sapphire substrate // J. Appl. Phys., 105, 063515 (2009).

5. V.P. Kladko, A.V. Kuchuk, N.V. Safryuk, V.F. Machulin, A.E. Belyaev, H. Hardtdegen, S.A. Vitusevich, Mechanism of strain relaxation by twisted nanocolumns revealed in $\mathrm{AlGaN} / \mathrm{GaN}$ heterostructures // Appl. Phys. Lett. 95, 031907 (2009).

6. V.P. Kladko, A.V. Kuchuk, P.M. Lytvyn, O.M. Yefanov, N.V. Safriuk, A.E. Belyaev, Yu.I. Mazur, E.A. DeCuir Jr, M.E. Ware, and G.J. Salamo, Substrate effects on the strain relaxation in GaN/AlN short-period superlattices // Nanoscale Res. Lett. 7, p. 289 (2012).

7. H. Amano, K. Hiramatsu, and I. Akasaki, Heteroepitaxial growth and the effect of strain on the luminescence properties of $\mathrm{GaN}$ films on $(11 \overline{2} 0)$ and (0001) sapphire substrates // Jpn. J. Appl. Phys., 27, L1384 (1988).

8. A. Bykhovski, B. Gelmont, and M. Shur, The influence of strain-induced electric field on the charge distribution in GaN-AlN-GaN structure // J. Appl. Phys. 74, p. 6734 (1993).

9. F. Bernardini, V. Fiorentini, and D. Vanderbilt, Spontaneous polarization and piezoelectric constant of III-V nitrides // Phys. Rev. B, 56, R10024 (1997).

10. P.K. Kandaswamy, F. Guillot, E. Bellet-Amalric, E. Monroy, L. Nevou, M. Tchernycheva, A. Michon, F.H. Julien, E. Baumann, F.R. Giorgetta, D. Hofstetter, T. Remmele, M. Albrecht, S. Birner, and Le Si Dang, GaN/AIN short-period superlattices for intersubband optoelectronics: A systematic study of their epitaxial growth, design, and performance // $\mathrm{J}$. Appl. Phys. 104, 093501 (2008).

11. P.K. Kandaswamy, C. Bougerol, D. Jalabert, P. Ruterana, and E. Monroy, Strain relaxation in 
short-period polar GaN/AlN superlattices // J. Appl. Phys. 106, 013526 (2009).

12. A. Sarua, Hangfeng Ji, M. Kuball, M.J. Uren, T. Martin, K.J. Nash, K.P. Hilton, and R.S. Balmer, Piezoelectric strain in AlGaN/GaN heterostructure field-effect transistors under bias // Appl. Phys. Lett. 88, 103502 (2006).

13. O.M. Yefanov, V.P. Kladko, V.F. Machulin, V.B. Molodkin, Dynamical Diffraction of X-rays in Multilayered Structures. Naukova Dumka, Kyiv, 2008.

14. P.F. Fewster, X-Ray Scattering from Semiconductors. London, Imperial College Press, 2000.

15. O.M. Yefanov, V.P. Kladko, The solution of the dispersion equation in an explicit format for a case of two strong waves // Metallofizika $i$ Noveishie Tekhnologii, 28, p. 227-244 (2006).

16. V.B. Molodkin, S.I. Olikhovskii, E.G. Len, E.N. Kislovskii, V.P. Kladko, O.V. Reshetnyk, T.P. Vladimirova, B.V. Sheludchenko, Sensitivity of triple-crystal X-ray diffractometers to microdefects in silicon // phys. status solidi $(a), \mathbf{2 0 6}(8)$, p. 17611765 (2009).

17. M. Yamaguchi, T. Yagi, T. Sota, T. Deguchi, K. Shimada, and S. Nakamura, Brillouin scattering study of bulk GaN // J. Appl. Phys. 85, p. 8502 (1999).

18. W. Paszkowicz, X-ray powder diffraction data for indium nitride // Powder Diffract., 14, p. 258 (1999).

19. H. Heinke, V. Kirchner, S. Einfeldt, A. Hommel, $\mathrm{X}$-ray diffraction analysis of the defect structure in epitaxial GaN // Appl. Phys. Lett. 77, p. 2145 (2000).
20. N.V. Safriuk, G.V. Stanchu, A.V. Kuchuk, V.P. Kladko, A.E. Belyaev, V.F. Machulin, X-ray diffraction investigation of $\mathrm{GaN}$ layers on $\mathrm{Si}(111)$ and $\mathrm{Al}_{2} \mathrm{O}_{3}(0001)$ substrates // Semiconductor Physics, Quantum Electronics \& Optoelectronics, 16(3), p. 265-272 (2013).

21. Yu.P. Pershin, V.A. Sevtyukova, Ye.N. Zubarev, A.S. Oberemok, V.P. Melnyk, B.M. Romanyuk, V.G. Popov, P.M. Lytvyn, Investigation of the phase interfaces in periodic multilayer $\mathrm{Mo} / \mathrm{Si}$ structures, using the method of mass-spectrometry of neutral particles // Metallofizika $i$ Noveishie Tekhnologii, 35(12), p. 1617-1627 (2013).

22. M.A. Moram, M.E. Vickers, X-ray diffraction of III-nitrides // Repts. Progr. Phys. 72, 036502 (2009).

23. V. Srikant, J.S. Speck, D.R. Clarke, Mosaic structure in epitaxial thin films having large lattice mismatch // J. Appl. Phys. 82, p. 4286 (1997).

24. R.N. Kyutt, M.P. Shcheglov, V.Yu. Davydov, A.S. Usikov, Deformation of layers in superlattices AlGaN/GaN according to XRD analysis // Fizika Tverd. Tela, 46, p. 353 (2004), in Russian.

25. Y. Kotsar, B. Doisneau, E. Bellet-Amalric, A. Das, E. Sarigiannidou, and E. Monroy, Strain relaxation in $\mathrm{GaN} / \mathrm{Al}_{\mathrm{x}} \mathrm{Ga}_{1-\mathrm{x}} \mathrm{N}$ superlattices grown by plasma molecular-beam epitaxy // J. Appl. Phys. 110, 033501 (2011).

26. A.V. Kuchuk, V.P. Kladko, T.L. Petrenko, V.P. Bryksa, A.E. Belyaev, Yu.I. Mazur, M.E. Ware, E.A. DeCuir Jr, and G.J. Salamo, Mechanism of strain-influenced quantum well thickness reduction in GaN/AlN short-period superlattices // Nanotechnology, 25, 245602 (2014). 